\title{
A Formal Analysis of Information Disclosure in Data Exchange
}

\author{
Gerome Miklau and Dan Suciu \\ Computer Science and Engineering \\ University of Washington \\ \{gerome,suciu\}@cs.washington.edu
}

\begin{abstract}
We perform a theoretical study of the following queryview security problem: given a view $V$ to be published, does $V$ logically disclose information about a confidential query $S$ ? The problem is motivated by the need to manage the risk of unintended information disclosure in today's world of universal data exchange. We present a novel information-theoretic standard for query-view security. This criterion can be used to provide a precise analysis of information disclosure for a host of data exchange scenarios, including multi-party collusion and the use of outside knowledge by an adversary trying to learn privileged facts about the database. We prove a number of theoretical results for deciding security according to this standard. We also generalize our security criterion to account for prior knowledge a user or adversary may possess, and introduce techniques for measuring the magnitude of partial disclosures. We believe these results can be a foundation for practical efforts to secure data exchange frameworks, and also illuminate a nice interaction between logic and probability theory.
\end{abstract}

\section{INTRODUCTION}

Traditional security mechanisms protect data at the physical level. For example, firewalls and other perimeter mechanisms prevent the release of raw data, as do conventional access controls for file systems and databases. In data exchange, however, such mechanisms are limited since they can only protect the data up to the first authorized recipient. When data is exchanged with multiple partners, information may be unintentionally disclosed, even when all physical protection mechanisms work as intended. As an extreme example, Sweeney proved this [19] when she retrieved the privileged medical data of William Weld, former governor of the state of Massachusetts, by linking information from two publicly available databases, each of which was considered

Permission to make digital or hard copies of all or part of this work for personal or classroom use is granted without fee provided that copies are not made or distributed for profit or commercial advantage, and that copies bear this notice and the full citation on the first page. To copy otherwise, to republish, to post on servers or to redistribute to lists, requires prior specific permission and/or a fee.

SIGMOD 2004 June 13-18, 2004, Paris, France.

Copyright 2004 ACM 1-58113-859-8/04/06 . . \$ \$5.00. secure in isolation ${ }^{1}$.

We address the case when data originates at a single source, but may be exchanged with multiple partners and further disseminated. To prevent unintended information disclosure, we need a formalism to protect the data at the logical level. Specifically, we study the following fundamental problem, called the query-view security problem: given views $V_{1}, V_{2}, \ldots$ that we want to publish, do they logically disclose any information about a query $S$ that we want to keep secret? The views are expressed in a query language, and may remove data items through projections or selections, or break associations between data items. Adopting the nomenclature of the cryptographic community we say that Alice wants to give Bob the views $V_{1}, V_{2}, \ldots$ over a public channel, but would like to prevent an adversary Mallory (or even Bob) from learning the answer to secret query $S$. The query expressions $S, V_{1}, V_{2}, \ldots$ are known by the adversary, the answers to $V_{1}, V_{2}, \ldots$ are published, while the underlying database remains secret.

In many cases a disclosure may not reveal a fact with complete certainty or an exact answer to a query. Consider a database consisting of a single relation:

\section{Employee(name,department,phone)}

Imagine Alice publishes the view projecting on (name, department) to Bob, and publishes the view projecting (department, phone) to Carol.

$$
\begin{aligned}
V_{\text {Bob }} & =\Pi_{\text {name,department }}(\text { Employee }) \\
V_{\text {Carol }} & =\Pi_{\text {department }, \text { phone }}(\text { Employee })
\end{aligned}
$$

Alice wants to protect employees' phone numbers, so the secret query would be:

$$
S=\Pi_{\text {name }, \text { phone }}(\text { Employee })
$$

If Bob and Carol collude, they cannot compute the answer to $S$ since the association between name and phone

\footnotetext{
${ }^{1}$ A voter registration list for governor Weld's home city included the voters' names, zip, birth-date, and sex. The state's Group Insurance Commission published a database containing "anonymized" medical data, including only the patients' zip, birth-date, and sex, but omitting the name. Only one person matched governor Weld's zip, birth-date, and sex, allowing Sweeney to retrieve his medical records.
} 
is not present in $V_{B o b}$ and $V_{C a r o l}$. However a partial disclosure has occurred, and it is a potentially serious threat to the security of $S$. For example, if only four people work in each department then an adversary can guess any person's phone number with a $25 \%$ chance of success by trying any of the four phone numbers in her department.

For a more complex example, consider a manufacturing company that needs to exchange XML messages with several partners. Each message type is a dynamic view that computes on request some information about the company's manufacturing data: $V_{1}$ contains detailed information about parts for a specific product, to be exchanged with suppliers; $V_{2}$ contains detailed information about products' features, options, and selling prices, to be exchanged with retailers and customers; while $V_{3}$ provides labor cost information to be sent to a tax consulting firm. The company wants to keep secret the internal manufacturing cost for its products, which can be expressed in terms of a query $S$. Conventional protection mechanisms can ensure that each message is received and read only by authorized users, but are powerless beyond that point. At a minimum, the company would like to audit each of the three views and ensure that it doesn't disclose the secret information to its authorized recipient. But in addition the company would like to understand if any information is disclosed when views are combined (colluded), for example when $V_{3}$ is accidentally or intentionally sent to a supplier, or when the tax consulting firm merges with one of the customers. None of these tasks are performed today, manually or automatically, because there is no clear understanding of when and how much information is disclosed at the logical level.

Our study of logical information disclosure applies directly to the following data exchange scenarios:

Multi-party collusion Alice would like to publish $n$ views $V_{1}, \ldots V_{n}$ to $n$ different users. Given a secret query $S$, which are the multiple party collusions among the users that will violate the confidentiality of $S$ ?

Prior Knowledge Suppose Mallory, the adversary, has some knowledge about the database represented in the form of a query $K$. Can Alice publish $V$ without disclosing anything about the secret query $S$ ? The prior knowledge may be common knowledge, such as the fact that social security numbers are unique or that phone numbers with the same area code are in the same state, or may be more specific knowledge about the domain that Mallory has acquired somehow.

Relative security Alice has already published a view $U$. This already leaked some information about a secret query $S$, but was considered an acceptable risk by Alice. Now she wishes to publish an additional view $V$. Does $V$ disclose any additional information about $S$ over what was already disclosed by $U$ ? This is not the same as saying that the query $S$ is secure with respect to the pair of views $U, V$, because $S$ is not secure w.r.t. $U$.
Our first contribution is a formal definition of queryview security that captures the disclosure of partial information. Inspired by Shannon's notion of perfect secrecy [18], the definition compares the likelihood of an adversary guessing the answer to secret query $S$ with and without knowledge of views $V_{1}, V_{2}, \ldots V_{n}$. When the difference is zero, we say that the query is secure w.r.t. the views. To the best of our knowledge this is the first attempt to formalize logical information disclosure in databases. Our second contribution consists of a number of theoretical results about query-view security: we prove a necessary and sufficient condition for query-view security, and show that the security problem for conjunctive queries is $\Pi_{2}^{p}$-complete; we generalize query-view security to account for pre-existing knowledge; and when the query is not secure w.r.t. a view, we characterize the magnitude of disclosure. These theoretical results illuminate an interesting connection between logic and probability theory.

\section{Examples of information disclosure}

Table 1 contains a set of query-view pairs referring to an Employee relation, along with an informal description of the information the views disclose about the query. These examples represent a spectrum of information disclosure, beginning with total disclosure, and ending with a secure query and view. The first query and view is an obvious example of a total disclosure because $S_{1}$ is answerable using $V_{1}$.

Example (2) is precisely the example mentioned above in which Bob (given $V_{2}$ ) and Carol (given $V_{2}^{\prime}$ ) collude to cause a partial disclosure. It is worth noting that a contained rewriting of $S_{2}$ using $V_{2}, V_{2}^{\prime}$ exists here. For small departments, it may be easy for Mallory to guess the association between names and phone numbers.

As another example of partial disclosure, consider example (3), whose view is $V_{3}=\Pi_{\text {name }}$ (Employee). We ask whether query $S_{3}=\Pi_{\text {phone }}$ (Employee) is secure when this view is published. In this case the view omits phone entirely and would seem to reveal nothing about phone numbers in $S_{3}$. Surprisingly, the view does disclose some information about the secret query. In particular, it can reveal something about the size of the Employee relation, and therefore contains some small amount of information about the omitted column. We describe this further in Section 3.

The last example, Table 1(4), is a case where no information is disclosed. The names of employees in the Admin department reveal nothing about the names of employees in the Shipping department.

\section{Insufficiency of related techniques}

The query-view security problem is superficially-related to a number of other database problems. We briefly review some of them here and explain why they do not solve the query-view security problem.

Query answering The basic problem addressed in query answering [13] is: given a view $V$ (or several such views), 


\begin{tabular}{|c|c|c|c|c|}
\hline & View $(s)$ & Query & $\begin{array}{l}\text { Information } \\
\text { Disclosure }\end{array}$ & $\begin{array}{l}\text { Query- } \\
\text { View } \\
\text { Security }\end{array}$ \\
\hline (1) & $V_{1}(n, d):-\operatorname{Employee}(n, d, p)$ & $S_{1}(d):-\operatorname{Employee}(n, d, p)$ & Total & No \\
\hline$(2)$ & $\begin{array}{l}V_{2}(n, d):-\operatorname{Employee}(n, d, p) \\
V_{2}^{\prime}(d, p):-\operatorname{Employee}(n, d, p)\end{array}$ & $S_{2}(n, p):-\operatorname{Employee}(n, d, p)$ & Partial & No \\
\hline (3) & $V_{3}(n):-\operatorname{Employee}(n, d, p)$ & $S_{3}(p):-\operatorname{Employee}(n, d, p)$ & Minute & No \\
\hline (4) & $V_{4}(n):-\operatorname{Employee}(n$, "Admin", $p)$ & $S_{4}(n):-\operatorname{Employee}(n$, "Shipping", $p)$ & None & Yes \\
\hline
\end{tabular}

Table 1: Pairs of views and queries, over relation Employee(name, department, phone) and an informal description of their information disclosure.

answer a query $S$ by using only the data in the view(s). A more refined version, called query rewriting, asks for the answer to be given as a query over the view(s) $V$. The connection to our problem is that, whenever $S$ can be answered from $V$, then $S$ is obviously not secure, as in Table 1(1). However, adopting non-answerability as a criterion for security would clearly be a mistake: it would classify example (2) as secure. As we claim above, even though the query $S$ may be not answerable using $V$, substantial information about the query may be revealed, allowing an attacker to guess the secret information with a high probability of success.

A related strategy considers a view $V$ and its answer $v=V(I)$ as a constraint on the set of possible database instances, making some impossible. The set of possible answers to a query $S$ given $V$ may therefore be reduced. (In some cases this set may have size 1 in which case the answer to $S$ is determined by $v=V(I)$.) We might say $S$ is secure given $V$ if every possible answer to $S$ remains possible given $V$. This criterion would classify Examples (2) and (3) correctly, that is it would capture the partial disclosures in these cases, but not in others. However, this standard of security ignores the likelihood of the possible answers of $S$. While $V$ may not rule out any possible answers to $S$, some answers may become less likely, or in the extreme, virtually improbable without contradicting the security criterion. In such a situation, we should say that $V$ does in fact contain substantial information for answering $S$. Our definition of queryview security handles this case.

Statistical database security A statistical database publishes views consisting of aggregate functions over a subset of records. Information is thus hidden by aggregating data, and the security problem is to ensure that data in individual tuples remains secret. The security of statistical databases has a long history $[1,6]$. Our work on query-view security is orthogonal: we do not hide data by aggregation, but rather through projections or selections, or breaking associations. Rather than statistics, we need to use probability theory to reason about query-view security.

The organization of the paper is as follows. Section 2 presents notation and a probabilistic model of databases. Section 3 describes our definition of query-view security and its main results. Section 4 extends these results to include prior knowledge. Section 5 presents techniques for measuring disclosure when security fails. We summarize related work and conclude in Sections 6 and 7 . Omitted proofs are included in [16].

\section{BACKGROUND AND NOTATION}

As we mentioned, many disclosures do not involve an adversary computing $S$ completely according to standard database query semantics. Instead a partial disclosure reveals to Mallory something about the likelihood of answers to a secret query $S$. After an overview of notation, we present our security model that allows formal statements about the probability of a database and query answer. Our discussion is based on the relational model.

\subsection{Basic Notations}

We assume a standard relational schema consisting of several relation names $R_{1}, R_{2}, \ldots$, each with a set of attribute names. Let $D$ be the finite domain, which includes all values that can occur in any attributes in any of the relations. For example $D$ may be the set of decimal numbers up to an upper bound, and all strings up to a given length. In a particular setting we may consider further restricting $D$, e.g. to include only valid disease names, valid people names, or valid phone numbers.

We use datalog notation to denote tuples belonging to the relations of the given schema. For example $R_{1}(a, b, c)$ denotes a tuple in $R_{1}$, and $R_{3}(b, a, a)$ denotes a tuple in $R_{3}$. Let $\operatorname{tup}(D)$ be the set of all tuples over all relations in the schema that can be formed with constants from the domain $D$. A database instance $I$ is any subset of $\operatorname{tup}(D)$, and we denote by $\operatorname{inst}(D)$ the set of all database instances over the domain $D$. A query of arity $k$ is a function $Q: \operatorname{inst}(D) \rightarrow \mathcal{P}\left(D^{k}\right)$. For an instance $I$, $Q(I)$ denotes the result of applying $Q$ to $I$. A boolean query is a query of arity 0 . A monotone query has the property $I \subseteq I^{\prime} \Rightarrow Q(I) \subseteq Q\left(I^{\prime}\right)$. In most of the paper our discussion will focus on conjunctive queries with 
inequalities, written in datalog notation. For example:

$Q(x):-R_{1}(x, a, y), R_{2}(y, b, c), R_{3}(x,-,-), x<y, y \neq c$

Here $x, y$ are variables, - are anonymous variables (each occurrence of - is distinct from all others) and $a, b, c$ are constants.

\subsection{The Security Model}

We assume a probability distribution on the tuples, $\mathbf{P}$ : $\operatorname{tup}(D) \rightarrow[0,1]$, s.t. for each $t_{i} \in \operatorname{tup}(D), \mathbf{P}\left(t_{i}\right)=x_{i}$ represents the probability that the tuple $t_{i}$ will occur in a database instance. We will refer to the pair $(D, \mathbf{P})$ as a dictionary. A dictionary induces a probability distribution on specific instances: for any $I \in \operatorname{inst}(D)$, the probability that the database instance is precisely $I$ is:

$$
\mathbf{P}[I]=\prod_{t_{i} \in I} x_{i} \cdot \prod_{t_{j} \notin I}\left(1-x_{j}\right)
$$

For example, if the schema consists of a single table Patient(name, disease) representing sensitive data in a hospital, then the domain $D$ may consist of all possible names (e.g. those occurring in a phone book for the entire country), together with all possible diseases cataloged by the CDC. For each tuple $t_{i}=\operatorname{Patient}($ name, disease), $\mathbf{P}\left(t_{i}\right)$ is the (very small) probability that a person with that name and that disease is in the hospital's database. To illustrate, assuming $10^{8}$ distinct names and 500 distinct diseases ${ }^{2}$ there are $n=5 \times 10^{10}$ tuples in $\operatorname{tup}(D)$, and one possible probability distribution is $\mathbf{P}\left(t_{i}\right)=200 / n$ for every $t_{i} \in \operatorname{tup}(D)$. This is a uniform probability distribution, for which the expected database size is 200 tuples. A more accurate, but far more complex probability distribution is one that takes into account the different risk factors of various ethnic groups and for each diseases. For example the probability of a tuple Patient( "John Johnson", "Cardiovascular Disease") will be slightly higher than the probability of the tuple Patient( "Chen Li", "Cardiovascular Disease"), if Americans have a higher risk of a Cardiovascular Disease than Chinese, and the nationality of John Johnson is likely to be American while that of Chien Li is likely to be Chinese.

The probability $\mathbf{P}\left(t_{i}\right)$ may be too complex to compute in practice, but computing it is not our goal. Instead we will assume that Mallory can compute it, and can use it to derive information about the secret query $S$. Thus, we endow the adversary with considerable power, and study under which circumstances no information is disclosed.

Given a probability distribution over database instances, a query $S$ attains some answer $s$ with probability equal to the sum of the probabilities of the satisfying instances:

$$
\mathbf{P}[S(I)=s]=\sum_{\{I \in \operatorname{inst}(D) \mid S(I)=s\}} \mathbf{P}[I]
$$

Remark In our model the tuples are independent probabilistic events. This is a limitation. In practice, the

${ }^{2}$ Fewer than 500 are listed at
http://www.cdc.gov/health/.

occurrence of tuples may be correlated due to underlying relationships in the data or integrity constraints. If tuples are positively correlated (respectively, negatively correlated) the presence of one tuple increases (decreases) the likelihood of another. For example, a key constraint introduces strong negative correlations. We will address some of these limitations in Section 4 by studying query-view security relative to some prior knowledge expressing a functional dependency. However, extending our results to a model that can capture arbitrary correlations between tuples remains open.

\section{QUERY-VIEW SECURITY}

In this section we formalize our notion of query-view security, describe its basic properties, and state our main theorems which result in a decision procedure for queryview security.

\subsection{Definition}

Our standard for query-view security is inspired by Shannon's definition of perfect secrecy [18]. Let $\bar{V}=V_{1}, \ldots, V_{k}$ be a set of views, and $S$ a "secret" query. Both the views and the query are computed over an instance $I$ of a relational schema. We consider an adversary, Mallory, who is aware of the domain and probability distribution over instances (the dictionary), and is given $\bar{V}(I)$ (but not $I)$. Mallory's objective is to compute $S(I)$. The definition below captures the intuition that $\bar{V}(I)$ discloses no information about $S(I)$. Below, $\bar{V}(I)=\bar{v}$ means $V_{1}(I)=v_{1} \wedge \ldots \wedge V_{k}(I)=v_{k}$.

Definition 3.1 (Query-View Security). Let $(D, \mathbf{P})$ be a dictionary. A query $S$ is secure w.r.t. a set of views $\bar{V}$ if for any possible answer s to the query, and any possible answers $\bar{v}$ to the views, the following holds:

$$
\mathbf{P}[S(I)=s]=\mathbf{P}[S(I)=s \mid \bar{V}(I)=\bar{v}]
$$

Query-view security is denoted $\left.S\right|_{\mathbf{P}} V$, or simply $S \mid V$ if $\mathbf{P}$ is understood from the context.

The left hand side of equation (3) represents the a priori probability that $S$ attains a particular answer $s$ over the instance $I$, which can be computed by Mallory using $(D, \mathbf{P})$. The right hand side is also the probability that $S(I)=s$ but conditioned on the fact that $\bar{V}(I)=\bar{v}$. The security condition asserts the equality of these two probabilities (for all possible $s, \bar{v}$ ) and therefore says that nothing beyond the a priori knowledge is provided by $\bar{V}$. Equation (3) is also the familiar definition of independence of two statistical events. Accordingly, $S$ is secure w.r.t. $\bar{V}$ iff $S$ and $\bar{V}$ are statistically independent events. We can rewrite (3) as follows:

$\mathbf{P}[S(I)=s] \mathbf{P}[\bar{V}(I)=\bar{v}]=\mathbf{P}[S(I)=s \wedge \bar{V}(I)=\bar{v}]$

Next we apply the definition in two examples:

Example 3.2 Non-security Consider a single relation $R(X, Y)$ and domain $D=\{a, b\}$. There are 4 possible 
tuples $R(a, a), R(a, b), R(b, a), R(b, b)$, and the set of instances inst $(D)$ contains the 16 subsets of these. Assume for simplicity that $\mathbf{P}\left(t_{i}\right)=1 / 2$ for each tuple $t_{i}$, and consider the following query and view:

$$
\begin{aligned}
V(x) & :-R(x, y) \\
S(y) & :-R(x, y)
\end{aligned}
$$

$V$ projects the first attribute of $R$ while $S$ projects the second. Although we might expect that the view provides no information about the query, it is actually not the case that $S \mid V$. Informally, the answer to $V$ contains some information about the size of the database which impacts answers to $S$. Consider a particular answer $\{(\mathrm{a})\}$ for $S$. There are 3 equally-likely instances generating this answer: $\{R(a, a)\},\{R(b, a)\}$, and $\{R(a, a), R(b, a)\}$. Therefore, we have a priori probability:

$$
\mathbf{P}[S(I)=\{(a)\}]=3 / 16
$$

Now suppose we are given that $V(I)=\{(b)\}$. There are again 3 instances, only one of which causes $S(I)=$ $\{(a)\}$, so because each instance is equally-likely we have:

$$
\mathbf{P}[S(I)=\{(a)\} \mid V(I)=\{(b)\}]=1 / 3
$$

This contradicts (3) for the particular answers considered, and it follows that $S$ and $V$ are not secure for this particular probability distribution. We show in the next section that they are not secure for any distribution.

Example 3.3 Security As an example of a secure query and view, consider the same schema and dictionary, and:

$$
\begin{aligned}
& V(x):-R(x, b) \\
& S(y):-R(y, a)
\end{aligned}
$$

Here $S$ is secure w.r.t. $V$. We prove this later, but illustrate here with one example. Consider one possible output of $S: S(I)=\{(a)\}$. There are 4 instances that lead to this output, $\{R(a, a)\},\{R(a, a), R(a, b)\}$, $\{R(a, a), R(b, b)\}$, and $\{R(a, a), R(a, b), R(b, b)\}$, hence:

$$
\mathbf{P}[S(I)=\{(a)\}]=4 / 16=1 / 4
$$

Consider also one possible output of $V$, say $V(I)=$ $\{(b)\}$. There are four instances $I$ satisfying this constraint: $\{R(b, b)\},\{R(b, b), R(a, a)\},\{R(b, b), R(b, a)\}$, $\{R(b, b), R(a, a), R(b, a)\}$. Of these only one also results in $S(I)=\{(a)\}$, hence:

$$
\mathbf{P}[S(I)=\{(a)\} \mid V(I)=\{(b)\}]=1 / 4
$$

One can manually check, for all possible combinations of outputs of $S$ and $V$, that the probability of $S$ is unchanged by publishing $V$. We will provide an easier criterion for checking this shortly.

\section{Discussion}

Several properties of query-view security follow, providing intuition and justifying our choice of definition.

Reflexivity It follows from Bayes' Theorem that security is a reflexive relation: $S \mid \bar{V}$ iff $\bar{V} \mid S$.

Security (not obscurity) We always assume that publishing the views $\bar{V}$ includes exposing both the view definitions and their answers over the hidden database. Basing the security on concealing the view and query expressions is dangerous. We thus avoid the pitfall of "security by obscurity", identified long ago by the cryptographic community as ineffective [17].

Instance-independence If the query $S$ is secure w.r.t. the views $\bar{V}$, it remains so even if the underlying database instance $I$ changes: this follows from the fact that Eq.(3) must hold for any query output $s$ and any view outputs $\bar{v}$. We say that query-view security is instance independent. This property is necessary in applications like message-based data exchange, were messages are exchanged continuously, even as the database changes. Once $S \mid \bar{V}$ has been checked, the views $\bar{V}(I)$ can safely be exchanged without any compromise of $S(I)$. In fact, one can prove that if successive instances are independent from one another, then even if Mallory collects snapshots of the views at various moments of time, $\bar{V}\left(I_{1}\right)$, $\bar{V}\left(I_{2}\right), \ldots, \bar{V}\left(I_{t}\right)$, he still cannot learn anything about any of $S\left(I_{1}\right), \ldots, S\left(I_{t}\right)$. This way of defining security is different from the standard definition in statistical databases. There the security criteria often apply to a particular database instance, and may fail if the instance is later updated. For example, one security criterion requires that the aggregate function be computed only on cells that are "large enough". One data instance may be secure, but it becomes insecure when tuples are deleted (making some cells too small), or when tuples are inserted (creating new cells, which are small).

Dictionary-independence The definition of queryview security $S \mid \bar{V}$ is for a particular dictionary $(D, \mathbf{P})$. In practice, however, the dictionary is often ill-defined: for example the probability distribution $\mathbf{P}$ is impossible to compute, and even the domain $D$ may be hard to define precisely. Thus, we are interested in a stronger version of security, which is dictionary-independent. Our results in the next section provide necessary and sufficient conditions for dictionary-independent security. They show, surprisingly, that, in some cases, security for some dictionary implies security for all dictionaries (see Theorem 3.8 and Proposition 3.9).

Collusions Given $\bar{V}=V_{1}, \ldots V_{k}$, we will show in Theorem 3.5 that $S \mid \bar{V}$ if and only if $S \mid V_{i}$ for all $i=1, \ldots, k$. This has the following consequence. If we send different views to different users, but have determined that the secret query $S$ is secure w.r.t. each view separately, then nothing will be leaked about $S$ even if the recipients collude, i.e. exchange the views they received and try to learn something about $S$ by examining all the views together. This strong property is a consequence of our adoption of a notion of perfect secrecy to define security. Disclosure through collusion happens when each view leaks very little information when taken separately, but together may leak a lot of information about $S$. We will re-examine collusion in Sec. 5 when we discuss measuring disclosures.

Query answering The database community has studied extensively the following query answering problem. 
Given a set of views $\bar{V}=V_{1}, \ldots V_{k}$ and another view $V^{\prime}$ find a function $f$ s.t. for any instance $I, V^{\prime}(I)=$ $f(\bar{V}(I))$ : in this case we say that $V^{\prime}$ is answerable from $\bar{V}$. In the related query rewriting problem, $f$ is restricted to be expressed in a query language. It is natural to ask about the relationship to security. Intuitively, if $V^{\prime}$ is answerable from $\bar{V}$, then the information content of $V^{\prime}$ is not more than that of $\bar{V}$, and any query $S$ which is secure w.r.t. $\bar{V}$ should be also secure w.r.t. to $V^{\prime}$. This intuition is correct, and can be proven formally ${ }^{3}$. A similar result holds when security fails: if $\neg(S \mid \bar{V})$ and another query $S^{\prime}$ is computable from $S$, then $\neg\left(S^{\prime} \mid \bar{V}\right)$.

Aggregates When applied to queries with aggregates our definition of security results in a very strict condition: no query and view containing an aggregate over a common tuple are secure. Techniques from statistical databases are better-suited for the case of queries with aggregates, and are orthogonal to our discussion. We therefore omit aggregate functions from the query language we consider.

\subsection{Fundamental Theorems of Query-View Security}

At this point, the only obvious procedure for deciding query-view security is to compute probabilities for each answer to the query and view. In addition to the computational complexity of this strategy, it requires recomputation for each dictionary. In this subsection we present techniques for deciding query-view security by analyzing the query and view definitions, and prove that this technique is dictionary-independent.

Definition 3.4 (CRitical tuple). Let $D$ be a finite domain and $Q$ be a query. A tuple $t \in \operatorname{tup}(D)$ is critical for $Q$ if there exists an instance $I \in \operatorname{inst}(D)$ such that $Q(I-\{t\}) \neq Q(I)$. The set of critical tuples of $Q$ is denoted $\operatorname{crit}_{D}(Q)$, or simply crit $(Q)$ when $D$ is understood from the context.

The intuition is that $t$ is critical for $Q$ if there exists some instance where dropping $t$ makes a difference.

For a simple illustration, consider the boolean query $Q():-R\left(a_{1}, x\right)$ and let $D=\left\{a_{1}, \ldots, a_{n}\right\}$. Any tuple of the form $R\left(a_{1}, a_{i}\right), i=1, \ldots, n$, is critical for $Q$, because $Q$ returns true on the database consisting of the single tuple $R\left(a_{1}, a_{i}\right)$, but if we remove that tuple then we get the empty database on which the query returns false.

We can now formulate the characterization of queryview security. The proof is in Sec. 3.3.

Theorem 3.5. Let $D$ be a domain. Let $S$ be a query

$\overline{{ }^{3} \text { Since } \mathbf{P}\left[V^{\prime}(I)=v^{\prime}\right]=} \sum_{\bar{v}}\left\{\mathbf{P}[\bar{V}(I)=\bar{v}] \mid f(\bar{v})=v^{\prime}\right\}$ and $\mathbf{P}\left[S(I)=s \wedge V^{\prime}(I)=v^{\prime}\right]=\sum_{\bar{v}}\{\mathbf{P}[S(I)=s \wedge \bar{V}(I)=\bar{v}] \mid$ $\left.f(\bar{v})=v^{\prime}\right\}$, which implies that the view $V^{\prime}$ satisfies Equation (4). In particular, if $V$ is a boolean view, then it follows that $S \mid V$ iff $S \mid \neg V$. and $\bar{V}$ be a set of views. Then $\left.S\right|_{\mathbf{P}} \bar{V}$ for every probability distribution $\mathbf{P}$ iff $\operatorname{crit}_{D}(S) \cap \operatorname{crit}_{D}(\bar{V})=\emptyset$.

Here $\operatorname{crit}(\bar{V})$ is $\operatorname{crit}\left(V_{1}\right) \cup \ldots \cup \operatorname{crit}\left(V_{k}\right)$. In particular it follows that $\left.S\right|_{\mathbf{P}} \bar{V}$ forall $\mathbf{P}$ iff $\left.S\right|_{\mathbf{P}} V_{i}$ forall $i=1, \ldots, k$ and forall $\mathbf{P}$. The theorem says that the only way a query can be insecure w.r.t. some views is if they have some common critical tuple. This result translates the probabilistic definition of query-view security into a purely logical statement, which does not involve probabilities. This is important, because it allows us to reason about query-view security by using traditional techniques from database theory and finite model theory.

Next we revisit the query and view examples from the last section and apply Theorem 3.5.

Example 3.6 In Example 3.2, we saw that security fails to hold for $V(x):-R(x, y)$ and $S(y):-R(x, y)$. Every tuple is critical for $V$ : for example, $R(a, b)$ is critical for $V$ because $V(\{\})=\{\}$ while $V(\{R(a, b)\})=$ $\{(a)\}$. Similarly, every tuple is critical for $S$, so because $\operatorname{crit}(V) \cap \operatorname{crit}(S)$ is nonempty, we conclude $\neg\left(\left.S\right|_{\mathbf{P}} V\right)$ at least for some probability distribution $\mathbf{P}$.

Example 3.7 We argued in Example 3.3 that security holds for $V(x):-R(x, b)$ and $S(y):-R(y, a)$. The critical tuples of $S$ are $\operatorname{crit}(S)=\{R(a, a), R(b, a)\}$, and similarly $\operatorname{crit}(V)=\{R(a, b), R(b, b)\}$. Because $\operatorname{crit}(S) \cap$ $\operatorname{crit}(V)=\emptyset$, Theorem 3.5 allows us to conclude $\left.S\right|_{\mathbf{P}} V$ for every probability distribution $\mathbf{P}$.

So far $S$ and $\bar{V}$ were allowed to be arbitrary queries. We now restrict $S$ and $\bar{V}$ to be monotone queries, and will prove that the definition of query-view security is, for all practical purposes, dictionary-independent. The main step is the following theorem, whose proof is in Sec. 3.3.

Theorem 3.8 (Probability-IndePEndence). Let $D$ be a domain, and $S, \bar{V}$ be any monotone queries. Let $\mathbf{P}_{0}$ be a probability distribution s.t. $\forall t, \mathbf{P}_{0}(t) \neq 0$ and $\mathbf{P}_{0}(t) \neq 1$. If $S \mid \mathbf{P}_{0} \bar{V}$ then for every probability distribution $\mathbf{P},\left.S\right|_{\mathbf{P}} \bar{V}$.

This is a surprising theoretical result, which says that if a query is secure even for one probability distribution, then it is secure for all such distributions. Continuing Example 3.2, both $S$ and $V$ are monotone. It follows that $\neg\left(\left.S\right|_{\mathbf{P}} V\right)$ for any probability distribution $\mathbf{P}$ which is $\neq 0$ and $\neq 1$. Notice that for the trivial distribution $\mathbf{P}(t)=1, \forall t$, we have $\left.S\right|_{\mathbf{P}} V$, because in this case the answer to both $S$ and $V$ are known.

We still need to show that the definition is insensitive to a particular choice of domain, and for that we will further restrict all queries to be conjunctive queries. As 
we vary the domain $D$, we will always assume that $D$ includes all the constants occurring in $S$ and $\bar{V}$.

Proposition 3.9 (Domain-Independence). Let $n$ be largest number of variables and constants occurring in any of the conjunctive queries $S, V_{1}, \ldots, V_{k}$. If there exists a domain $D_{0}$ s.t. $\left|D_{0}\right| \geq n(n+1)$, and crit $_{D_{0}}(S) \cap$ crit $_{D_{0}}(\bar{V})=\emptyset$, then for any domain D, s.t. $|D| \geq$ $n(n+1), \operatorname{crit}_{D}(S) \cap \operatorname{crit}_{D}(\bar{V})=\emptyset$.

We now discuss how to decide query-view security for conjunctive queries $S$ and $\bar{V}$. Our goal is to check dictionary-independent security, hence we need to check whether $\operatorname{crit}_{D}(S) \cap \operatorname{crit}_{D}(\bar{V})=\emptyset$, and we assume that the domain $D$ is "large enough". The previous proposition gives us an exponential time algorithm: pick a domain $D_{0}$ with $n(n+1)$ constants, then enumerate exhaustively all instances $I \subseteq D_{0}$ and tuples $t \in I$, checking whether $t$ is a critical tuple for $S$, and for $\bar{V}$. This also shows that the query-view security problem is in complexity class $\Pi_{2}^{p}$.

Deciding query-view security is also $\Pi_{2}^{p}$-hard. We can prove that checking $t \notin \operatorname{crit}(Q)$ is $\Pi_{2}^{p}$-hard: this is a non-trivial result, whose proof uses a lengthy reduction from the $\forall \exists 3$-CNF problem, and is omitted. Instead, we illustrate with an example why computing $\operatorname{crit}(Q)$ is non-obvious. Clearly, any critical tuple $t$ must be an homomorphic image of some subgoal of $Q$. But the following example shows the converse is not true:

$$
Q():-R(x, y, z, z, u), R(x, x, x, y, y)
$$

Consider the tuple $t=R(a, a, b, b, c)$, which is a homomorphic image of the first subgoal. Yet $t$ is not critical. Indeed, let $I$ be any database s.t. $Q(I)=$ true. Then the first subgoal must be mapped to $t$. But that means that both $x$ and $y$ are mapped to $a$. Thus the second subgoal must be mapped to the tuple $t^{\prime}=R(a, a, a, a, a)$ and then $t^{\prime} \in I$. Then the first subgoal can also be mapped to $t^{\prime}$, hence $t$ is not critical.

Next, we show that deciding whether $\operatorname{crit}(S) \cap \operatorname{crit}(V)=$ $\emptyset$ is at least as hard as deciding whether a tuple $t$ is not critical for a query $Q$. Indeed, if we define $V=Q$, and $S($ ): $-t$ (i.e. $S$ simply checks for the presence of the tuple $t)$, then $t \notin \operatorname{crit}(Q)$ iff $\operatorname{crit}(S) \cap \operatorname{crit}(V)=\emptyset$. For the former we have claimed that it is $\Pi_{2}^{p}$-hard. In summary:

THEOREM 3.10. The problem of deciding whether a conjunctive query $S$ is secure w.r.t. to a set of conjunctive views $V_{1}, \ldots V_{k}$ is $\Pi_{2}^{p}$-complete (query complexity).

\footnotetext{
${ }^{4}$ For conjunctive queries without order predicates it suffices to pick the domains $D_{0}, D$ with size $\geq n$. When order predicates are allowed, then we need $\bar{n}$ fresh constants between any two constants mentioned in the queries, which leads to $n(n+1)$.

${ }^{5}$ Recall that NP is the class of problems that can be expressed as $\{z \mid \exists y \phi(y, z)\}$ where the "certificate" $y$ has length polynomial in $z$ and $\phi$ is PTIME computable. Complexity class $\Pi_{2}^{p}$ consists of problems that can be expressed as $\{z \mid \forall x \exists y \phi(x, y, z)\}$ where $x, y$ are polynomial in $z$ and $\phi$ is PTIME computable.
}

A practical algorithm For practical purposes, one can check $\operatorname{crit}(S) \cap \operatorname{crit}(\bar{V})=\emptyset$ and hence $S \mid \bar{V}$ quite efficiently. Simply compare all pairs of subgoals from $S$ and from $\bar{V}$. If any pair of subgoals unify, then $\neg S \mid \bar{V}$. While false positives are possible, they are rare: this simple algorithm would correctly classify all examples in this paper.

\subsection{Proof of the Fundamental Theorems}

The technique used in the proof of Theorems 3.5 and 3.8 is of independent interest, and we present it here. It may be skipped at a first reading. Throughout this subsection we will fix the domain $D$ and denote the set of tuples with $\operatorname{tup}(D)=\left\{t_{1}, \ldots, t_{n}\right\}$. Recall our notation from Sec. 2: $x_{1}=\mathbf{P}\left[t_{1}\right], \ldots, x_{n}=\mathbf{P}\left[t_{n}\right]$. Hence, a probability distribution $\mathbf{P}$ is given by a set of numbers $\bar{x} \in[0,1]^{n}$.

\section{The Boolean Case, Single View}

We first prove both theorems for the case of boolean queries; moreover, we will consider a single view, rather than a set of views. Given a boolean query $Q$, we denote by $\mathbf{P}[Q]$ the probability that $Q$ is true on a randomly chosen database instance. Recall from Equations (1) and (2) that this probability is given by:

$$
\begin{aligned}
\mathbf{P}[Q] & =\sum_{\{I \in \text { inst }(D) \mid Q(I)=\text { true }\}} \mathbf{P}[I] \\
\mathbf{P}[I] & =\prod_{t_{i} \in I} x_{i} \cdot \prod_{t_{j} \notin I}\left(1-x_{j}\right)
\end{aligned}
$$

Therefore $\mathbf{P}[Q]$ is given by a polynomial in the variables $x_{1}, \ldots, x_{n}$, which we denote $f_{Q}\left(x_{1}, \ldots, x_{n}\right)$ or $f_{Q}(\bar{x})$.

Example 3.11 Let $D=\{a, b\}$, and consider the boolean query:

$$
Q():-R(a, x), R(x, x)
$$

In this case $\operatorname{tup}(D)=\left\{t_{1}, t_{2}, t_{3}, t_{4}\right\}$, where $t_{1}=R(a, a)$, $t_{2}=R(a, b), t_{3}=R(b, a)$, and $t_{4}=R(b, b)$. Then $Q$ can be written as the following DNF formula:

$$
Q=t_{1} \vee\left(t_{2} \wedge t_{4}\right)
$$

To compute $f_{Q}$ one enumerates all 16 database instances $I \subseteq \operatorname{tup}(D) . Q$ is true on 12 of them: $\left\{t_{2}, t_{4}\right\},\left\{t_{2}, t_{3}, t_{4}\right\}$, .... For each of them we apply Eq.(5). This results in a sum of 12 expressions:

$$
f_{Q}=\left(1-x_{1}\right) x_{2}\left(1-x_{3}\right) x_{4}+\left(1-x_{1}\right) x_{2} x_{3} x_{4}+\ldots
$$

After simplification we obtain: $f_{Q}=x_{1}+x_{2} x_{4}-x_{1} x_{2} x_{4}$. Let $Q^{\prime}:-R(b, a)$ (so that $f_{Q^{\prime}}=x_{3}$ ), and consider the boolean formula $Q \wedge Q^{\prime}$. The polynomial $f_{Q \wedge Q^{\prime}}$ is equal to $f_{Q} \times f_{Q^{\prime}}$, i.e. $\left(x_{1}+x_{2} x_{4}-x_{1} x_{2} x_{4}\right) x_{3}$ because $Q$ and $Q^{\prime}$ depend on disjoint sets of tuples.

Before we prove the two theorems we notice that queryview security for boolean queries can be restated as follows. Given boolean queries $S$ and $V,\left.S\right|_{\mathbf{P}} V$ iff:

$$
f_{S \wedge V}(\bar{x})=f_{S}(\bar{x}) \times f_{V}(\bar{x})
$$


where $\bar{x}$ corresponds to $\mathbf{P}$. Indeed, this represents precisely Equation (4) for one specific choice of $s$ and $v$, namely $s=$ true and $v=$ true. One can show that if Eq.(4) holds for (true, true), then it also holds for the other three combinations, (false,true), (true, false), (false, false). Thus, $\left.S\right|_{\mathbf{P}} V$ holds precisely if (6) holds.

We now restate the two Theorems for the boolean case:

Theorem $3.5 \forall \bar{x} \in[0,1]^{n} \cdot f_{S \wedge V}(\bar{x})=f_{S}(\bar{x}) \times f_{V}(\bar{x})$ iff $\operatorname{crit}_{D}(S) \cap \operatorname{crit}_{D}(V)=\emptyset$.

Theorem 3.8 If $S$ and $V$ are monotone boolean queries, then $\exists \bar{x} \in(0,1)^{n} \cdot f_{S \wedge V}(\bar{x})=f_{S}(\bar{x}) \times f_{V}(\bar{x})$ implies $\forall \bar{x} \in[0,1]^{n} \cdot f_{S \wedge V}(\bar{x})=f_{S}(\bar{x}) \times f_{V}(\bar{x})$.

The crux of the proof relies on a close examination of the polynomials $f_{Q}$. The properties we need are summarized below. Their proofs are straightforward and are omitted:

Proposition 3.12. Let $f_{Q}=\mathbf{P}[Q]$, where $Q$ is a boolean formula in $t_{1}, \ldots, t_{n}$. Then $f_{Q}$ is a polynomial in the variables $x_{1}, \ldots, x_{n}$ with the following properties:

1. For each $i=1, \ldots, n$, the degree of $x_{i}$ is $\leq 1$.

2. For each $i=1, \ldots, n$, the degree of $x_{i}$ is 1 iff $t_{i} \in \operatorname{crit}_{D}(Q) . \quad\left(\right.$ In Example 3.11, $\operatorname{crit}_{D}(Q)=$ $\left\{t_{1}, t_{2}, t_{4}\right\}$ and indeed $x_{1}, x_{2}, x_{4}$ have degree 1 , while $x_{3}$ has degree 0.$)$

3. If $\operatorname{crit}_{D}\left(Q_{1}\right) \cap \operatorname{crit}_{D}\left(Q_{2}\right)=\emptyset$ then $f_{Q_{1} \wedge Q_{2}}=f_{Q_{1}} \times f_{Q_{2}}$.

4. Choose values in $[0,1]^{n-1}$ for all variables except for one, $x_{i}: f_{Q}$ becomes a polynomial of degree $\leq 1$ in $x_{i}$. Then, if $Q$ is a monotone boolean formula, the coefficient of $x_{i}$ is $\geq 0$. In Example 3.11, the coefficient of $x_{4}$ in $f_{Q}$ is $x_{2}-x_{1} x_{2}$, which is always $\geq 0$ when $x_{1}, x_{2} \in[0,1]^{2}$.

5. Let $Q_{0}$ be the boolean formula obtained from $Q$ by setting $t_{n}=$ false, and $Q_{1}$ be the boolean formula obtained by setting $t_{n}=$ true. Then $f_{Q_{0}}=$ $f_{Q}\left[x_{n}=0\right]$ and $f_{Q_{1}}=f_{Q}\left[x_{n}=1\right]$. In example 3.11, $Q_{0}=t_{1}$ and $f_{Q}\left[x_{4}=0\right]=x_{1}$; similarly $Q_{1}=t_{1} \vee t_{2}$ and $f_{Q}\left[x_{4}=1\right]=x_{1}+x_{2}-x_{1} x_{2}$.

We prove now Theorem 3.5 for the boolean case. Assume first that $\operatorname{crit}_{D}(S) \cap \operatorname{crit}_{D}(V)=\emptyset$. Then $f_{S \wedge V}=$ $f_{S} \times f_{V}$, by Proposition 3.12, item 3. Assume now that $\forall \bar{x} \in[0,1]^{n} \cdot f_{S \wedge V}(\bar{x})=f_{S}(\bar{x}) \times f_{V}(\bar{x})$ holds. Then the polynomials $f_{S \wedge V}$ and $f_{S} \times f_{V}$ must be identical. In particular, $f_{S}$ and $f_{V}$ cannot have a common variable $x_{i}$, otherwise its degree would be 2 . Hence $\operatorname{crit}_{D}(S)$ and $\operatorname{crit}_{D}(V)$ cannot have a common tuple (Prop. 3.12 item 2).

Next we prove Theorem 3.8 for the boolean case. Consider the polynomial $g_{S, V}=f_{S \wedge V}-f_{S} \times f_{V}$. We show by induction on the number $n$ of tuples in $\operatorname{tup}(D)$ that $\forall \bar{x} \in[0,1]^{n}, g_{S, V}(\bar{x}) \geq 0$. It holds trivially for $n=$ 0 . For $n>0, g_{S, V}$ is a polynomial of degree $\leq 2$ in $x_{n}$, and the coefficient of $x_{n}^{2}$ is negative: this follows from Proposition 3.12 item 4 and the fact that $S, V$ are monotone. For $x_{n}=0$, the polynomial in $n-1$ variables $g_{S, V}\left[x_{n}=0\right]$ corresponds to the boolean formulas $S\left[t_{n}=\right.$ false $], V\left[t_{n}=\right.$ false $]$ (item 5 of the proposition), hence we can apply the induction hypothesis and obtain that $g_{S, V} \geq 0$ for $x_{n}=0$. Similarly, $g_{S, V} \geq 0$ for $x_{n}=1$, since now it corresponds to the boolean formulas $S\left[t_{n}=\right.$ true $], V\left[t_{n}=\right.$ true $]$. Furthermore, since $g_{S, V}$ has degree $\leq 2$ and the coefficient of $x_{n}^{2}$ is $\leq 0$, it follows that $g_{S, V} \geq 0$ for every $x_{n} \in[0,1]$. This completes the inductive proof. Now assume that for some $\bar{x} \in(0,1)^{n}$, $g_{S, V}(\bar{x})=0$. We will prove that $\operatorname{crit}_{D}(S) \cap \operatorname{crit}_{D}(V)=\emptyset$. Assume by contradiction that $t_{i} \in \operatorname{crit}_{D}(S) \cap \operatorname{crit}_{D}(V)$ for some tuple $t_{i}$. Then $g_{S, V}$ is a polynomial of degree 2 in $x_{i}$, with a negative coefficient for $x_{i}^{2}$, which has at least one root in $(0,1)$. It follows that $g_{S, V}$ must be $<0$ either in $x_{i}=0$, or in $x_{i}=1$, contradicting the fact that $g_{S, V} \geq 0$ for all $\bar{x} \in[0,1]^{n}$.

\section{The Boolean Case, Multiple Views}

Let $\bar{V}=V_{1}, \ldots, V_{n}$ be $n$ boolean views. The next step is to show that $\left.S\right|_{\mathbf{P}} \bar{V}$ forall $\mathbf{P}$ iff $\left.S\right|_{\mathbf{P}} V_{i}$ forall $i=1, \ldots, n$ and all P. We illustrate for $n=2$. For the 'only if' direction we prove Eq.(4) directly. To show $\mathbf{P}[S(I)=$ $\left.s \wedge V_{2}(I)=v_{2}\right]=\mathbf{P}[S(I)=s] \times \mathbf{P}\left[V_{2}(I)=v_{2}\right]$ we notice:

$$
\begin{aligned}
\mathbf{P}\left[S(I)=s \wedge V_{2}(I)=v_{2}\right] & =\sum_{v_{1}} \mathbf{P}\left[S(I)=s \wedge V_{1}(I)=v_{1} \wedge V_{2}(I)=v_{2}\right] \\
\mathbf{P}\left[V_{2}(I)=v_{2}\right] & =\sum_{v_{1}} \mathbf{P}\left[V_{1}(I)=v_{1} \wedge V_{2}(I)=v_{2}\right]
\end{aligned}
$$

then we use $\left.S\right|_{\mathbf{P}}\left(V_{1}, V_{2}\right)$. For the 'if' direction, we need to check $\mathbf{P}\left[S(I)=s \wedge V_{1}(I)=v_{1} \wedge V_{2}(I)=v_{2}\right]=\mathbf{P}[S(I)=$ $s] \times \mathbf{P}\left[V_{1}(I)=v_{1} \wedge V_{2}(I)=v_{2}\right]$. Using Theorem 3.5 for the boolean, single view case, it suffices to check $\operatorname{crit}_{D}(S) \cap \operatorname{crit}_{D}(V)=\emptyset$ where $V(I)$ is the boolean query $V_{1}(I)=v_{1} \wedge V_{2}(I)=v_{2}$. This follows from $\operatorname{crit}_{D}(V) \subseteq$ $\operatorname{crit}_{D}\left(V_{1}\right) \cup \operatorname{crit}_{D}\left(V_{2}\right)$, and the assumption, $\operatorname{crit}_{D}(S) \cap$ $\operatorname{crit}_{D}\left(V_{i}\right)=\emptyset$ for $i=1,2$.

\section{The Non-boolean Case}

We now generalize to non-boolean queries. Given a $k$ ary query $Q$, let $t_{1}, \ldots, t_{m}$ be all $k$-tuples over the domain $D\left(m=|D|^{k}\right)$. For each $i=1, \ldots, m$, define $Q_{i}^{b}$ the boolean query $Q_{i}^{b}(I)=\left(t_{i} \in Q(I)\right)$; that is, it checks whether $t_{i}$ is in $Q$. Notice that $\operatorname{crit}_{D}(Q)=$ $\bigcup_{i} \operatorname{crit}_{D}\left(Q_{i}^{b}\right)$, and if $Q$ is monotone then $Q_{i}^{b}$ is monotone for $i=1, \ldots, m$.

Given a domain $D$ and probability distribution, the following is easy to check, by applying directly the Definition 3.1. For any query $S$ and views $\bar{V}=V_{1}, \ldots V_{k}$ :

$$
\left.S\right|_{\mathbf{P}} \bar{V} \text { iff } \forall i, j, l .\left.S_{i}^{b}\right|_{\mathbf{P}} V_{j, l}^{b}
$$

Here $V_{j, l}^{b}$ denotes $\left(V_{j}\right)_{l}^{b}$. This immediately reduces both theorems to the boolean case.

\section{MODELING PRIOR KNOWLEDGE}

So far we have assumed that the adversary has no knowledge about the data other than the domain $D$ and the probability distribution $\mathbf{P}$ provided by the dictionary. Next we consider security in the presence of prior knowledge, which we denote with $K$. Our standard for security compares Mallory's knowledge about the secret 
query $S$ before and after publishing the views $\bar{V}$, but always assuming he knows $K$. In the most general case $K$ is any boolean statement on the database instance $I$. For example it can be a key or foreign-key constraint, some previously published views, or some general knowledge about the domain. $K$ is thus any boolean predicate on the instance $I$, and we write $K(I)$ whenever $I$ satisfies $K$. To avoid introducing new terminology, we will continue to call $K$ a boolean query. We do not however restrict $K$ by requiring that it be expressed in a particular query language.

\subsection{Definition and Main Theorem}

As before we assume domain $D$ to be fixed. $K$ is a boolean query, while $S$ and $\bar{V}$ are arbitrary queries.

Definition 4.1 (Prior Knowledge Security). Let $\mathbf{P}$ be a probability distribution on the tuples. We say that $S$ is secure w.r.t. $\bar{V}$ under prior knowledge $K$ if for every $s, \bar{v}$ :

$$
\mathbf{P}[S(I)=s \mid K(I)]=\mathbf{P}[S(I)=s \mid \bar{V}(I)=\bar{v} \wedge K(I)]
$$

We denote prior knowledge security by $K:\left.S\right|_{\mathbf{P}} \bar{V}$.

Applying Bayes' theorem reduces the above to:

$$
\begin{array}{r}
\mathbf{P}[S(I)=s \wedge \bar{V}(I)=\bar{v} \wedge K(I)] \times \mathbf{P}[K(I)]= \\
\quad \mathbf{P}[S(I)=s \wedge K(I)] \times \mathbf{P}[\bar{V}(I)=\bar{v} \wedge K(I)]
\end{array}
$$

Both the prior knowledge and the relative security applications mentioned in Sec. 1 are modeled as a security problem with prior knowledge. In the case of relative security, we take $K$ to be the knowledge that the prior view has some given answer.

Theorem 3.5, which showed query-view security is equivalent to disjointness of the critical tuples, can be generalized for security with prior knowledge. We state the theorem for the boolean case, and will discuss specific generalizations to non-boolean queries and views.

ThEOREM 4.2. Let $D$ be a domain, $T=\operatorname{tup}(D)$, and $K, S, V$ be arbitrary boolean queries. Then $K:\left.S\right|_{\mathbf{P}} V$ for all probability distributions $\mathbf{P}$ iff the following holds:

COND-K There exists sets of tuples $T_{1}, T_{2}$ and boolean queries $K_{1}, K_{2}, V_{1}, S_{2}$ s.t.:

$$
\begin{aligned}
& T_{1} \cap T_{2}=\emptyset \\
& K=K_{1} \wedge K_{2} \\
& S \wedge K=K_{1} \wedge S_{2} \\
& V \wedge K=V_{1} \wedge K_{2} \\
& \operatorname{crit}_{D}\left(K_{1}\right) \subseteq T_{1} \quad \operatorname{crit}_{D}\left(K_{2}\right) \subseteq T_{2} \\
& \operatorname{crit}_{D}\left(V_{1}\right) \subseteq T_{1} \quad \operatorname{crit}_{D}\left(S_{2}\right) \subseteq T_{2}
\end{aligned}
$$

Informally, the theorem says that the space of tuples can be partitioned into $T_{1}$ and $T_{2}$ such that property $K$ is the conjunction of two independent properties, $K_{1}$ over $T_{1}$ and $K_{2}$ over $T_{2}$. In addition, assuming $K$ holds, $S$ just says something about the tuples in $T_{2}$ (and nothing more about $T_{1}$ ). Similarly, when $K$ holds, $V$ just says something about $T_{1}$ (and nothing more about $T_{2}$ ).

By itself, this theorem does not result in a practical decision procedure, because it is too general. We show, however, how it can be applied to specific applications, and in particular derive decision procedures.

\subsection{Applying Prior Knowledge}

Application 1: No prior knowledge As a baseline check, let's see what happens if there is no prior knowledge. Then $K=$ true and condition COND-K says that there are two disjoint sets of tuples $T_{1}$ and $T_{2}$ such that $\operatorname{crit}_{D}(S) \subseteq T_{2}$ and $\operatorname{crit}_{D}(V) \subseteq T_{1}$. This is equivalent to saying $\operatorname{crit}_{D}(S) \cap \operatorname{crit}_{D}(V)=\emptyset$, thus we recover Theorem 3.5 for boolean queries.

Application 2: Keys and foreign keys The notion of query-view secrecy is affected by keys and foreignkeys constraints $K$. For an illustration, consider the boolean query: $S():-R(a, b)$, and the boolean view $V():-R(a, c)$. Here $a, b, c$ are distinct constants. We have $\left.S\right|_{\mathbf{P}} V$ for any $\mathbf{P}$, because $\operatorname{crit}_{D}(S)=\{R(a, b)\}$ and $\operatorname{crit}_{D}(V)=\{R(a, c)\}$ are disjoint. But now suppose that the first attribute of $R$ is a key. Then by knowing $V$ we know immediately that $S$ is false, which is a total information disclosure, hence $K: S \mid V$ does not hold.

We apply now Theorem 4.2 to derive a general criterion for query-view secrecy in the presence of key constraints $K$. Given a domain $D$, define the following equivalence relation on $\operatorname{tup}(D): t \equiv_{K} t^{\prime}$ if $t$ and $t^{\prime}$ are tuples over the same relation, and they have the same key. In the example above, we have $R(a, b) \equiv_{K} R(a, c)$, and $R(a, b) \not_{K} R(d, b)$ for a new constant $d$. Given a query $Q$, denote $\operatorname{crit}_{D}(Q, K)$ the set of tuples $t$ s.t. there exists a database instance $I$ that satisfies the key constraints $K$ and $Q(I) \neq Q(I-\{t\})$. The following criterion can be proven from Theorem 4.2 and shows how to check $K: S \mid \bar{V}$.

Corollary 4.3. Let $K$ be a set of key constraints, $D$ a domain, and $S, \bar{V}$ be any queries. Then $\left.S\right|_{\mathbf{P}} \bar{V}$ for any $\mathbf{P}$ iff $\forall t \in \operatorname{crit}_{D}(S, K), \forall t^{\prime} \in \operatorname{crit}_{D}(\bar{V}, K), t \neq_{K} t^{\prime}$. In particular, the problem whether $K:\left.S\right|_{\mathbf{P}} V$ for all $\mathbf{P}$ is decidable, and $\Pi_{2}^{p}$-complete.

As a simple illustration, in the previous example, we have $\operatorname{crit}_{D}(S, K)=\{R(a, b)\}$, $\operatorname{crit}_{D}(V, K)=\{R(a, c)\}$, and $R(a, b) \equiv_{K} R(a, c)$, hence it is not the case that $K$ : $\left.S\right|_{\mathbf{P}} V$ for all $\mathbf{P}$. Foreign keys can be handled similarly, however the corresponding decidability and complexity result holds only when the foreign keys introduce no cycles.

Application 3: Cardinality Constraint. What happens if Mallory has some partial knowledge about the cardinality of the secret database? This is quite common in practice. For example the number of patients in a hospital is likely to be between 100 or 1,000 , but 
not 2 and not $1,000,000$. In this case $K$ is a cardinality constraint, such as "there are exactly $n$ tuples in $I$ " or "there are at most $n$ tuples" or "at least $n$ tuples". Surprisingly, there are no secure queries when the prior knowledge involves any cardinality constraints! This follows from Theorem 4.2 since $\mathrm{K}$ cannot be expressed as $K_{1} \wedge K_{2}$ over disjoint sets of tuples, by a simple counting argument, except for the trivial case when $T_{1}=\emptyset$ or $T_{2}=\emptyset$. Hence, no query is perfectly secret w.r.t. to any view in this case, except if one of them $(S$ or $V)$ is trivially true or false.

Application 4: Protecting Secrets with Knowledge Sometimes prior knowledge can protect secrets! Take any queries $S, \bar{V}$, and assume that $S$ is not secure w.r.t. $\bar{V}$. Suppose now that we disclose publicly the status of every tuple in $\operatorname{crit}_{D}(S) \cap \operatorname{crit}_{D}(\bar{V})$. That is, for each common critical tuple $t$ we announce whether $t \in I$ or $t \notin I$. If we denote with $K$ this knowledge about all common critical tuples, then Theorem 4.2 implies that $K:\left.S\right|_{\mathbf{P}} \bar{V}$ for any $\mathbf{P}$, as we show below. For a simple illustration, assume $S():-R(a,-)$ and $V():-R(-, b)$. They are not secure because $\operatorname{crit}_{D}(S) \cap \operatorname{crit}_{D}(V)=$ $\{R(a, b)\}$. But now suppose we disclose that the pair $(a, b)$ is not in the database, $R(a, b) \notin I$, and call this knowledge $K$. Then $K:\left.S\right|_{\mathbf{P}} V$. The same is true if we publicly announce that $R(a, b)$ is in the database instance. We prove this formally next:

Corollary 4.4. Let $K$ be such that $\forall t \in \operatorname{crit}_{D}(S) \cap$ $\operatorname{crit}_{D}(\bar{V})$, either $K \models t \in I$, or $K \models t \notin I$. Then, for every $\mathbf{P}, K:\left.S\right|_{\mathbf{P}} \bar{V}$.

Proof. We will prove this for two boolean queries $S, V$ only: the general case follows easily. Let $T_{1}=$ $\operatorname{crit}_{D}(S) \cap \operatorname{crit}_{D}(V)$, and $T_{2}=\operatorname{tup}(D)-T_{1}$. Let $K_{1}=K$, $K_{2}=$ true, $S_{2}=S, V_{1}=V \wedge K$. Then the conditions of Theorem 4.2 are satisfied, hence $K:\left.S\right|_{\mathbf{P}} V$ for any P.

Application 5: Prior Views. Suppose Alice already published a view $U$ (there may have been leakage about $S$, but she decided the risk was acceptable). Now she wants to publish another view $V$, and she wonders: will I leak any more information about $S$ ?

Using Theorem 4.2 we give below a decision procedure for the case of conjunctive queries, but only when $U$ is a boolean query. This is a limitation, and due to the fact that both sides of the formula (7) are linear in $S$ and $\bar{V}$, but not in $K$ : this made it possible to generalize statements from boolean queries $S, V$ to arbitrary ones, but not for $K$. To simplify the statement, we also restrict $S$ and $V$ to be boolean: these, however, can be generalized to arbitrary conjunctive queries.

Corollary 4.5. Let $U, S, V$ be boolean conjunctive queries. Then $U:\left.S\right|_{\mathbf{P}} V$ for every probability distribu- tion $\mathbf{P}$ iff each of the queries can be split as follows:

$$
\begin{aligned}
U & =U_{1} \wedge U_{2} \\
S & =S_{1} \wedge S_{2} \\
V & =V_{1} \wedge V_{2}
\end{aligned}
$$

Such that the sets crit $_{D}\left(U_{1}\right) \cup \operatorname{crit}_{D}\left(S_{1}\right) \cup \operatorname{crit}_{D}\left(V_{1}\right)$ and $\operatorname{crit}_{D}\left(V_{2}\right) \cup$ crit $_{D}\left(S_{2}\right) \cup$ crit $_{D}\left(V_{2}\right)$ are disjoint, and $U_{1} \Rightarrow$ $S_{1}$ and $U_{2} \Rightarrow V_{2}$. Hence, $U:\left.S\right|_{\mathbf{P}} V$ is decidable.

The proof follows rather directly from Theorem 4.2 and is omitted. For a simple illustration consider:

$$
\begin{aligned}
U & :-R_{1}(a, b,-,-), R_{2}(d, e,-,-) \\
S & :-R_{1}(a,-,-,-), R_{2}(d, e, f,-) \\
V & :-R_{1}(a, b, c,-), R_{2}(d,-,-,-)
\end{aligned}
$$

Here $S$ is not secure w.r.t. either $U$ or $V$. However, $U$ : $S \mid V$. By giving out $U$ we already disclosed something about $S$, namely $R_{1}(a,-,-,-)$. By publishing $V$ in addition we do not further disclose any information.

\subsection{Proof of Theorem 4.2}

Proof. (sketch) For boolean queries, $K:\left.S\right|_{\mathbf{P}} V$ can be expressed as follows:

$$
\mathbf{P}[S \wedge V \wedge K] \times \mathbf{P}[K]=\mathbf{P}[S \wedge K] \times \mathbf{P}[V \wedge K]
$$

Using the notation $f_{Q}$ for a boolean query $Q$ (see Sec. 3.3), this becomes:

$$
f_{S \wedge V \wedge K}(\bar{x}) \times f_{K}(\bar{x})=f_{S \wedge K}(\bar{x}) \times f_{V \wedge K}(\bar{x})
$$

We need to prove that (8) holds for any $\bar{x} \in[0,1]^{n}$ iff COND-K holds. For that we need the properties of $f_{Q}$ in Proposition 3.12 plus three more. Call any multivariable polynomial $g(\bar{x})$ of degree $\leq 1$ in each variable a boolean polynomial if $\forall \bar{x} \in\{0,1\}^{n}, g(\bar{x})$ is either 0 or 1. Clearly, any polynomial $f_{Q}$ is a boolean polynomial.

\section{Proposition 4.6.}

1. If $g$ is a boolean polynomial then there exists a unique boolean formula $Q$ s.t. $g=f_{Q}$.

2. Let $Q$ be a boolean formula, and suppose $f_{Q}$ is the product of two polynomials $f_{Q}=g \times h$. Then there exists a constant $c \neq 0$ s.t. both $\mathrm{cg}$ and $\frac{1}{c} h$ are boolean polynomials.

3. If $f_{Q}=f_{Q_{1}} \times f_{Q_{2}}$ then $\operatorname{crit}_{D}\left(Q_{1}\right) \cap \operatorname{crit}_{D}\left(Q_{2}\right)=\emptyset$.

We can now prove the equivalence of (8) to COND-K. Assume (8) holds for every $\bar{x} \in[0,1]^{n}$, i.e. this is an identity of polynomials. Then $f_{K}$ divides $f_{S \wedge K} \times f_{V \wedge K}$. Hence $f_{K}=g \times h$ where $g$ divides $f_{S \wedge K}$ and $h$ divides $f_{V \wedge K}$. By Prop. 4.6 we can assume that $g, h$ are boolean, hence $f_{K}=f_{K_{1}} \times f_{K_{2}}$ for some boolean formulas $K_{1}, K_{2}$, and moreover we have $K=K_{1} \wedge K_{2}$ and $\operatorname{crit}_{D}\left(K_{1}\right) \cap \operatorname{crit}_{D}\left(K_{2}\right)=\emptyset$. Since $f_{K_{1}}$ divides $f_{S \wedge K}$, we can write the latter as $f_{S \wedge K}=f_{K_{1}} \times f_{S_{2}}$, for some boolean query $S_{2}$, which implies $S \wedge K=K_{1} \wedge S_{2}$. Similarly, $f_{K_{2}}$ divides $f_{V \wedge K}$, hence we can write the latter 
as $f_{V \wedge K}=f_{V_{1}} \times f_{K_{2}}$ for some query $V_{1}$. Finally, substituting in (8) and simplifying with $f_{K_{1}} \times f_{K_{2}}$ we get $f_{S \wedge V \wedge K}=f_{V_{1}} \times f_{S_{2}}$. It follows that $f_{V_{1}}$ and $f_{S_{2}}$ have no common variables, hence $\operatorname{crit}_{D}\left(V_{1}\right) \cap \operatorname{crit}_{D}\left(S_{2}\right)=\emptyset$. Define $T_{1}=\operatorname{crit}_{D}\left(K_{1}\right) \cup \operatorname{crit}_{D}\left(V_{1}\right)$ and $T_{2}=\operatorname{tup}(D)-T_{1}$. Then it follows that $\operatorname{crit}_{D}\left(K_{2}\right) \subseteq T_{2}$ and $\operatorname{crit}_{D}\left(S_{2}\right) \subseteq T_{2}$, completing the proof of COND-K.

For the other direction, assume COND-K is satisfied and let's prove (8). We have:

$$
\begin{aligned}
f_{S \wedge V \wedge K} & =f_{\left(K_{1} \wedge V_{1}\right) \wedge\left(K_{2} \wedge S_{2}\right)}=f_{K_{1} \wedge V_{1}} \times f_{K_{2} \wedge S_{2}} \\
f_{K} & =f_{K_{1}} \times f_{K_{2}} \\
f_{S \wedge K} & =f_{K_{1}} \times f_{S_{2} \wedge K_{2}} \\
f_{V \wedge K} & =f_{V_{1} \wedge K_{1}} \times f_{K_{2}}
\end{aligned}
$$

and (8) follows immediately.

\section{MEASURING DISCLOSURES}

Our standard for query-view security is very strong. It classifies as insecure query-view pairs that are considered secure in practice. In many applications we can tolerate deviations from this strong standard, as long as the deviations are not too large. We discuss here a measure of information disclosure that attempts to quantify the amount by which a query and view depart from our definition of security. Ours is one possible choice of measure; others are definitely possible. The main objective is to show that the theoretical concepts and results presented in this work can be employed to evaluate information disclosure in practical settings. We restrict our discussion to the case of no prior knowledge.

We will define a measure of positive information disclosure. This is easier to analyze, and far more important in practice than negative information disclosure. An example of the former is whether "John Johnson" has "cardiovascular disease"; and example of the latter is whether "John Johnson" does not have "cardiovascular disease". We will restrict the queries $S$ and $\bar{V}$ to be monotone queries, and will study atomic statements given by inclusions $s \subseteq S(I)$ and $\bar{v} \subseteq \bar{V}(I)$, which are monotone in $I$.

Our definition of leakage is the following:

$$
\operatorname{leak}(S, \bar{V})=\sup _{s, \bar{v}} \frac{\mathbf{P}[s \subseteq S(I) \mid \bar{v} \subseteq \bar{V}]-\mathbf{P}[s \subseteq S(I)]}{\mathbf{P}[s \subseteq S(I)]}
$$

The goal of a user wishing to publish $\bar{V}$ while not disclosing $S$ is to ensure that $\operatorname{leak}(S, \bar{V}) \ll 1$. This will ensure that $\mathbf{P}[s \subseteq S(I)]$ can increase only very little after publishing the view, giving Mallory a negligible amount of positive information. $\left.S\right|_{\mathbf{P}} \bar{V}$ iff $\operatorname{leak}(S, \bar{V})=0$.

For a given $s, \bar{v}$, denote $S_{s}(I)$ and $V_{\bar{v}}$ the boolean queries $s \subseteq S(I)$ and $\bar{v} \subseteq \bar{V}(I)$. Let $T_{s, \bar{v}}=\operatorname{crit}_{D}\left(S_{s}\right) \cap \operatorname{crit}_{D}\left(V_{\bar{v}}\right)$. We know from Theorem 3.5 that, when $T_{s, \bar{v}}=\emptyset$, then the difference in Eq.(9) is 0 for this pair of $s$ and $\bar{v}$. Our analysis of the leakage is based on the probability of $I$ having some tuple in $T_{s, \bar{v}}$. Let's denote $L_{s, \bar{v}}(I)$ the predicate $I \cap T_{s, \bar{v}} \neq \emptyset$, and $K_{s, \bar{v}}=\neg L_{s, \bar{v}}$. Then by Corollary 4.4, $K:\left.S_{s}\right|_{\mathbf{P}} V_{\bar{v}}$, and we can prove:
TheOREM 5.1. Suppose that there exists some $\varepsilon<1$ such that for all $s$ and for all $\bar{v}$ :

$$
\mathbf{P}\left[L_{s, \bar{v}}(I) \mid S_{s}(I) \wedge V_{\bar{v}}(I)\right]<\varepsilon
$$

Then:

$$
\operatorname{leak}(S, \bar{V}) \leq \frac{\varepsilon^{2}}{1-\varepsilon^{2}}
$$

Example 5.2 Minute leakage We will illustrate with the example in Table 1 . We abbreviate here with $E(n, d, p)$ the table Employee(name, department,phone). Consider the view $V(d):-E(n, d, p)$ and the query $S(n, p)$ : $-E(n, d, p)$ (it corresponds to $S_{2}$ in the table). To simplify the discussion we consider the case when $s$ and $v$ consists of a single tuple. First, $s=\left(d_{\text {name }}, d_{\text {phone }}\right)$ is a secret name-phone pair, and $v=\left(d_{d e p t}\right)$ is some department that we publish. The boolean queries are $V_{v}(I)=(v \in V(I))$ and $S_{s}(I)=(s \in S(I))$. We have $\operatorname{crit}_{D}\left(V_{v}\right)=\left\{\left(-, d_{\text {dept }},-\right)\right\}, \operatorname{crit}_{D}\left(S_{s}\right)=\left\{\left(d_{\text {name }},-\right.\right.$, $\left.\left.d_{\text {phone }}\right)\right\}$, and $T_{s, v}=\left\{\left(d_{\text {name }}, d_{\text {dept }}, d_{\text {phone }}\right)\right\}$. The conditional probability $\mathbf{P}\left[L \mid S_{s} \wedge V_{v}\right]$ is in this case $\mathbf{P}[L \wedge$ $\left.S_{s} \wedge V_{v}\right] / \mathbf{P}\left[S_{s} \wedge V_{v}\right]=\mathbf{P}[L] / \mathbf{P}\left[S_{s} \wedge V_{v}\right]$. This corresponds to $\varepsilon$ in Theorem 5.1. For example, assuming a uniform probability distribution $\mathbf{P}[t]=p$ for all $t \in t u p(D)$, and denoting $n_{1}, n_{2}, n_{3}$ the number of names, departments, and phone numbers in the dictionary, we have $\mathbf{P}[L]=p$ and $\mathbf{P}\left[S_{s} \wedge V_{v}\right]=p+\left(p\left(n_{2}-1\right)\right) \times\left(p\left(n_{1} n_{3}-\right.\right.$ $1)) \approx p^{2} n_{1} n_{2} n_{3}$. It follows that $\mathbf{P}\left[L \mid S_{s} \wedge V_{v}\right] \approx$ $1 /\left(p n_{1} n_{2} n_{3}\right)=1 / m$, where $m$ is the expected cardinality of an instance $I$. It can be shown that $\mathbf{P}\left[L \mid S_{s} \wedge V_{v}\right]$ is small for any sets $s, v$, implying that $\varepsilon$ in Theorem 5.1 is small. Hence the information disclosure about $S$ by publishing $V$ is minute. Example (3) in Table 1 can be explained similarly.

Example 5.3 Collusions Continuing the example, consider now the view $V(n, d):-E(n, d, p)$ and the query $S(n, p):-E(n, d, p)$ (they correspond to $S_{2}, V_{2}$ in the table). We will still restrict $s$ and $v$ to a single tuple. The interesting case here is when $s=\left(d_{\text {name }}, d_{\text {phone }}\right)$ and $v=\left(d_{\text {name }}, d_{\text {dept }}\right)$. As before we obtain $T_{s, v}=$ $\left\{\left(d_{\text {name }}, d_{\text {dept }}, d_{\text {phone }}\right)\right\}$. The conditional probability $\mathbf{P}[L \mid$ $\left.S_{s} \wedge V_{v}\right]=\mathbf{P}[L] / \mathbf{P}\left[S_{s} \wedge V_{v}\right]$ is slightly larger than in the previous example, because $\mathbf{P}\left[S_{s} \wedge V_{v}\right]$ has decreased. The $\varepsilon$ in Theorem 5.1 increases, suggesting more information disclosure. This is to be expected, since now the view discloses information about the names in the secret query.

Consider now the effect of the collusion between the view $V$ and the view $V^{\prime}(d, p):-E(n, d, p)$ (this is $V_{2}^{\prime}$ in Table 1). The interesting case to consider here is $s=$ $\left(d_{\text {name }}, d_{\text {phone }}\right), v=\left(d_{\text {name }}, d_{\text {dept }}\right), v^{\prime}=\left(d_{\text {dept }}, d_{\text {phone }}\right)$. We still have $T_{s, v, v^{\prime}}=\left\{\left(d_{\text {name }}, d_{\text {dept }}, d_{\text {phone }}\right)\right\}$. Now, $\mathbf{P}\left[L \mid S_{s} \wedge V_{v} \wedge V_{v^{\prime}}^{\prime}\right]=\mathbf{P}[L] / \mathbf{P}\left[S_{s} \wedge V_{v} \wedge V_{v^{\prime}}^{\prime}\right]$ is even smaller, because $\mathbf{P}\left[S_{s} \wedge V_{v} \wedge V_{v^{\prime}}^{\prime}\right]$ is smaller. The amount of leakage given by Theorem 5.1 is now larger.

\section{RELATED WORK}

We have made references in the body of the paper to both query answering [13] and statistical database security $[1,6]$. A probabilistic definition of security is 
discussed in $[2,3]$, with an emphasis on comparing probabilistic independence to algebraic independence.

The so-called FKG inequality [10] is a theoretical result about the correlation between events in a probability space. It is closely related to our security criterion, and can be used to show that $\mathbf{P}(V \wedge S) \geq \mathbf{P}(V) \mathbf{P}(S)$, for monotone boolean properties $V$ and $S$. However, it says nothing about when equality holds, and it's inductive proof offers little insight. Our Theorem 3.8 reproves this inequality and furthermore proves the necessary and sufficient condition for equality to hold. Another topic that connects logic to probability theory are the 0-1 laws [8,9], which hold for a logical language if, for each formula, the probability that a formula is true converges to either 0 or 1 as the size of the domain tends to infinity. Our definition of query-view security is not related to 0-1 laws: our domain size does not grow to infinity but remains fixed and we are concerned about the effect of one formula (the view) on another (the secret query).

Access control mechanisms in databases [4, 6] are used to define the rights of authorized subjects to read or modify data elements, and therefore usually offer control at a physical level, rather than a logical level. For example, a simple access control policy might prohibit access to confidential columns in a relation. This is similar to publishing a view after projecting out those columns. We have shown that such a view can in fact contain a partial disclosure about the confidential column (see Example 3.2).

A query is independent of an update if the application of the update cannot change the result of the query, for any state of the database. Detecting update independence is useful for maintenance of materialized views $[5,7,15]$ and efficient constraint checking [12]. Deciding whether a tuple $t$ is critical for a query $Q$ is equivalent to deciding whether $Q$ is independent of the update that deletes $t$ from the database. Update independence is undecidable for queries and updates expressed as datalog programs [15], but has been shown decidable for certain restricted classes of queries like conjunctive queries with comparisons $[5,7,15]$. The tight bounds shown in this paper for deciding $\operatorname{crit}(Q)$ constitute an interesting special case for update independence.

Finally, the goal of the probabilistic relational model $[11,14]$ is to model statistical patterns in huge amounts of data. The issues addressed are learning models from existing data, modeling statistics about a given database (e.g. to be used by a query optimizer), and inferring missing attribute values. These techniques do not provide us with means to reason about information disclosure, which is independent of a particular data instance.

\section{CONCLUSION}

We have presented a novel definition of security for analyzing the information disclosure of exchanged database views and shown several important results. Our work is foundational. However, we argue that it is indispens- able for developing practical tools for monitoring information disclosure. We have already shown how one of our foundational results (Corollary 4.4) can be applied to measure leakage (Theorem 5.1). We believe these results may be a basis for logically securing data exchange frameworks in the future.

Acknowledgments Ron Fagin, Venkatesan Guruswami, Daniele Micciancio, and Victor Vianu provided helpful comments on this work. Suciu was partially supported by the NSF CAREER Grant IIS-0092955, NSF Grant IIS-0140493, a gift from Microsoft, and a Sloan Fellowship. Miklau was partially supported by NSF Grant IIS-0140493.

\section{REFERENCES}

[1] N. R. Adam and J. C. Wortman. Security-control methods for statistical databases. ACM Computing Surveys, 21(4):515-556, Dec. 1989.

[2] F. Bancilhon and N. Spyratos. Protection of information in relational data bases. In $V L D B, 1977$.

[3] F. Bancilhon and N. Spyratos. Algebraic versus probabilistic independence in data bases. In PODS, pages 149-153, 1985.

[4] E. Bertino, S. Jajodia, and P. Samarati. Database security: research and practice. Inf. Syst., 20(7):537-556, 1995.

[5] J. A. Blakeley, N. Coburn, and P.-V. Larson. Updating derived relations: detecting irrelevant and autonomously computable updates. ACM Trans. Database Syst., 14(3):369-400, 1989.

[6] D. Denning. Cryptography and Data Security. Addison-Wesley Publishing Co., 1982.

[7] C. Elkan. Independence of logic database queries and update. In PODS, pages 154-160, 1990.

[8] R. Fagin. Probabilities on finite models. Notices of the Am. Math. Soc., October:A714, 1972.

[9] R. Fagin. Probabilities on finite models. Journal of Symbolic Logic, 41(1), 1976.

[10] C. Fortuin, P. Kasteleyn, and J. Ginibre. Correlation inequalities on some partially ordered sets. Comm.in Math. Physics, 22:89-103, 1971.

[11] L. Getoor, B. Taskar, and D. Koller. Selectivity estimation using probabilistic models. In SIGMOD, 2001.

[12] A. Gupta, Y. Sagiv, J. D. Ullman, and J. Widom. Constraint checking with partial information. In PODS, 1994.

[13] A. Halevy. Answering queries using views: A survey. VLDB Journal, 10(4):270-294, 2001.

[14] D. Koller and A. Pfeffer. Probabilistic frame-based systems. In Conference on Artificial Intelligence, pages 580-587, 1998 .

[15] A. Y. Levy and Y. Sagiv. Queries independent of updates. In Conference on Very Large Data Bases, pages 171-181, 1993.

[16] G. Miklau and D. Suciu. A formal analysis of information disclosure in data exchange. University of Washington Technical Report (TR 03-12-02), Dec 2003. www.cs.washington.edu/homes/gerome.

[17] B. Schneier. Applied Cryptography, Second Edition. John Wiley and Sons, Inc., 1996.

[18] C. E. Shannon. Communication theory of secrecy systems. In Bell System Technical Journal, 1949.

[19] L. Sweeney. $k$-Anonymity: a model for protecting privacy. Int. J. on Uncertainty, Fuzziness and Knowledge-based Systems, 10(5), 2002. 